\title{
Vascular anatomy in children with pulmonary hypertension regarding the transcatheter Potts shunt
}

\author{
Aleksander Sizarov, ${ }^{1}$ Francesca Raimondi, ${ }^{1,2}$ Damien Bonnet, ${ }^{1,3}$ \\ Younes Boudjemline ${ }^{1,3}$
}

\begin{abstract}
${ }^{1}$ Cardiologie pédiatrique, Centre de Référence Malformations Cardiaques Congénitales Complexes, Hôpital Universitair Necker Enfants Malades, Assistance Publique des Hôpitaux de Paris, Paris, France

${ }^{2}$ Service de Radiologie pédiatrique, Hôpital Universitair Necker Enfants Malades, Assistance Publique des Hôpitaux de Paris, Paris, France

${ }^{3}$ Université Paris V Descartes, Paris, France
\end{abstract}

\section{Correspondence to} Dr Younes Boudjemline, Cardiologie pédiatrique, Hôpital Necker Enfants Malades, 149 rue de Sèvres, Paris cedex 75015, France; younes.boudjemline@aphp.fr

Received 26 January 2016 Revised 31 March 2016 Accepted 17 May 2016 Published Online First 10 June 2016

\section{ABSTRACT \\ Objective To morphometrically characterise the region} of adjacent descending aorta (DAo) and left pulmonary artery (LPA) regarding the transcatheter creation of the reverse Potts shunt.

Methods and results Retrospective review of the invasive haemodynamic data and measurements of the vessel diameters, distances and angles based on the thoracic CT of children with idiopathic pulmonary arterial hypertension (PAH) with pulmonary-to-systemic systolic pressure ratio $\geq 0.5$. Forty-eight $\mathrm{CT}$ scans from 47 patients were analysed. Independent of the PAH severity, the diameters of DAo and LPA, and the area of tightest contact between these vessels were very similar in patients with either infrasystemic or isosystemic/ suprasystemic PAH. For total population, the tightest contact area (mean \pm SD, $51.8 \pm 31.9 \mathrm{~mm}^{2}$, range 12.5-177.7 $\mathrm{mm}^{2}$ ) had an elliptic shape stretched along the DAo length and LPA width. The shortest mean DAoLPA distance was $1.7 \pm 0.8 \mathrm{~mm}$ (range $1-5 \mathrm{~mm}$ ). Only one patient, from the suprasystemic PAH group, had the DAo-LPA distance $>4 \mathrm{~mm}$. None had lung tissue identified between these two vessels, while in four patients $(8.3 \%)$ the prominent bronchial artery was seen coursing exactly between the LPA and DAo. The difference of prevalence of the bronchial arteries between two vessels in patients with either infrasystemic $\mathrm{PAH}$ or isosystemic/suprasystemic PAH did not reach statistical significance.

Conclusions Children with idiopathic PAH showed no complicating anatomic or morphometric parameters of the region with adjacent DAo and LPA, which potentially determine the planning of the transcatheter creation of Potts shunt. It holds promises for standardisation of the procedure in the future.

\section{INTRODUCTION}

Idiopathic pulmonary arterial hypertension (PAH) is rare in children and has a poor prognosis due to progressive and inevitable development of the failure of the right ventricle (RV). ${ }^{1-3}$ Thus, elimination or delaying the onset of the RV failure is likely to improve survival in this condition. ${ }^{4}$ Patients with $\mathrm{PAH}$ due to Eisenmenger syndrome have significantly better survival and significantly lower incidence of the RV failure. ${ }^{5}$ These observations have led to an idea of creating an extracardiac right-to-left shunt in patients with the idiopathic suprasystemic PAH to decrease the afterload of the failing $\mathrm{RV}^{7}$ The surgical connection between the left pulmonary artery (LPA) and the descending aorta (DAo), the so-called Potts shunt, ${ }^{8}$ has been successfully used to achieve these goals ${ }^{9}$ and allowed prolonged survival and dramatic, long-lasting symptomatic improvement in children with drug-refractory $\mathrm{PAH} .{ }^{11}$ In our centre, the reverse Potts shunt is now considered as a first management step for the children with the failing $\mathrm{RV}$ due to severe suprasystemic PAH under maximal medical therapy.

Currently, the most used technique to create an unrestrictive extracardiac right-to-left shunt is the surgical direct LPA-to-DAo anastomosis. ${ }^{12}$ In rare cases of PAH associated with patent ductus arteriosus (PDA), the duct (even tiny) can be stented to allow unrestrictive right-to-left shunting. ${ }^{13-15}$ Furthermore, the vicinity of the DAo to LPA creates an attractive possibility to place a stent between the lumens of these two vessels creating an anastomosis percutaneously. ${ }^{16}{ }^{17}$ In this study, we sought to analyse the anatomic aspects determining the planning of the transcatheter Potts shunt in children with the idiopathic $\mathrm{PAH}$ and an eventual indication for such procedure.

\section{MATERIAL AND METHODS \\ Patients}

At our institution (Necker Hospital for Sick Children, Paris, France), patients with echocardiographic findings of $\mathrm{PAH}$ undergo a systematic evaluation, which includes invasive haemodynamic assessment and thoracic CT angiography to evaluate the pulmonary parenchyma and eventual thromboembolism. Database of our institution was reviewed to identify the patients with the idiopathic $\mathrm{PAH}$, and with the invasive haemodynamic data and CT scan available for analysis. Inclusion criterion was a pulmonary-to-systemic systolic arterial pressure ratio $\geq 0.5$ based on invasive measurements. Patients with the ratio $\geq 0.9$ were defined as having an isosystemic/suprasystemic PAH. Local ethical committee gave its approval for the retrospective review of the patient data.

\section{Morphometric measurements}

To standardise the measurements, the manipulations of the planes through all included scans were limited to the following steps. First, the axial plane was produced at the level of shortest DAo-LPA distance through the middle of the proximal LPA with its walls as parallel as possible, and the cross-section of the adjacent DAo as close to the circle as was feasible (figure 1A). Using the axial plane as reference, the oblique sagittal to coronal plane was produced through the middle of the DAo and
Raimondi $F$, Bonnet $D$, et al. Heart 2016;102:17351741. 
perpendicular to the adjacent LPA wall (figure 1B). In 12 scans (two from the same patient), a slight posterior angulation of the oblique plane was needed to obtain the parallel appearance of the walls of the DAo thoracic part and the cross-section of the adjacent LPA close to the circle. The resulted planes were used to measure the diameters, distances and angles considered important for the planning of the transcatheter creation of the Potts shunt. Diameters of LPA and DAo, and the shortest distance between the two vessels were measured in both planes on one line crossing the centre of either vessel and being perpendicular to the walls of adjacent one (figure 1C, D). The width and height of the tightest contact between LPA and DAo were defined as the part of the vessel circumferences with the shortest constant distance between adjacent walls, and used to calculate the estimation of the ellipse area. Furthermore, we measured two angles characterising further the spatial relationship between the LPA and DAo. In axial plane, the angle between the vertical line and the line perpendicular to the diameter measurements was defined as an angle of the right anterior oblique (RAO) angiographic projection producing the DAo and LPA shadows next to each other with minimal overlay (figure 1C). In oblique sagittal plane, the angle between the DAo longitudinal axis and the line of the diameter measurements was defined as an angle of the curve of catheter tip most facilitating the entrance of the perforating wire exactly through the centre of the tightest contact area between two vessels (figure 1D). To determine the intraobserver and interobserver variability, all above-mentioned measurements were repeated in 10 randomly selected patients by two investigators independently (AS and FR). Tissue surrounding the vessels was analysed as well.

\section{Three-dimensional reconstruction}

Serial CT angiogram images from the representative patient with suprasystemic PAH were used to prepare a three-dimensional (3D) reconstruction of the LPA and DAo. After loading of the images into the 3D reconstruction software Amira, V.5.2, the label-fields were manually created for the walls of the aorta and proximal aortic arch branches (colour-coded as red) and the main PA and its branches (colour-coded as purple). Additionally, the tracheobronchial lumen was labelled as light-green. After correcting deformities of the label-fields, the $3 \mathrm{D}$ surface was generated, simplified and further smoothed to obtain the final 3D model. By reconstructing the space between the adjacent walls of the LPA and DAo, we obtained the shape of the area of the tightest contact with the shortest constant distance between two vessels.

\section{Statistical analysis}

Mean values and their SDs are calculated where appropriate. The difference between the populations with either infrasystemic or suprasystemic PAH was tested using the Student's $\mathrm{t}$-distribution two-tailed test. A p value of 0.05 was considered
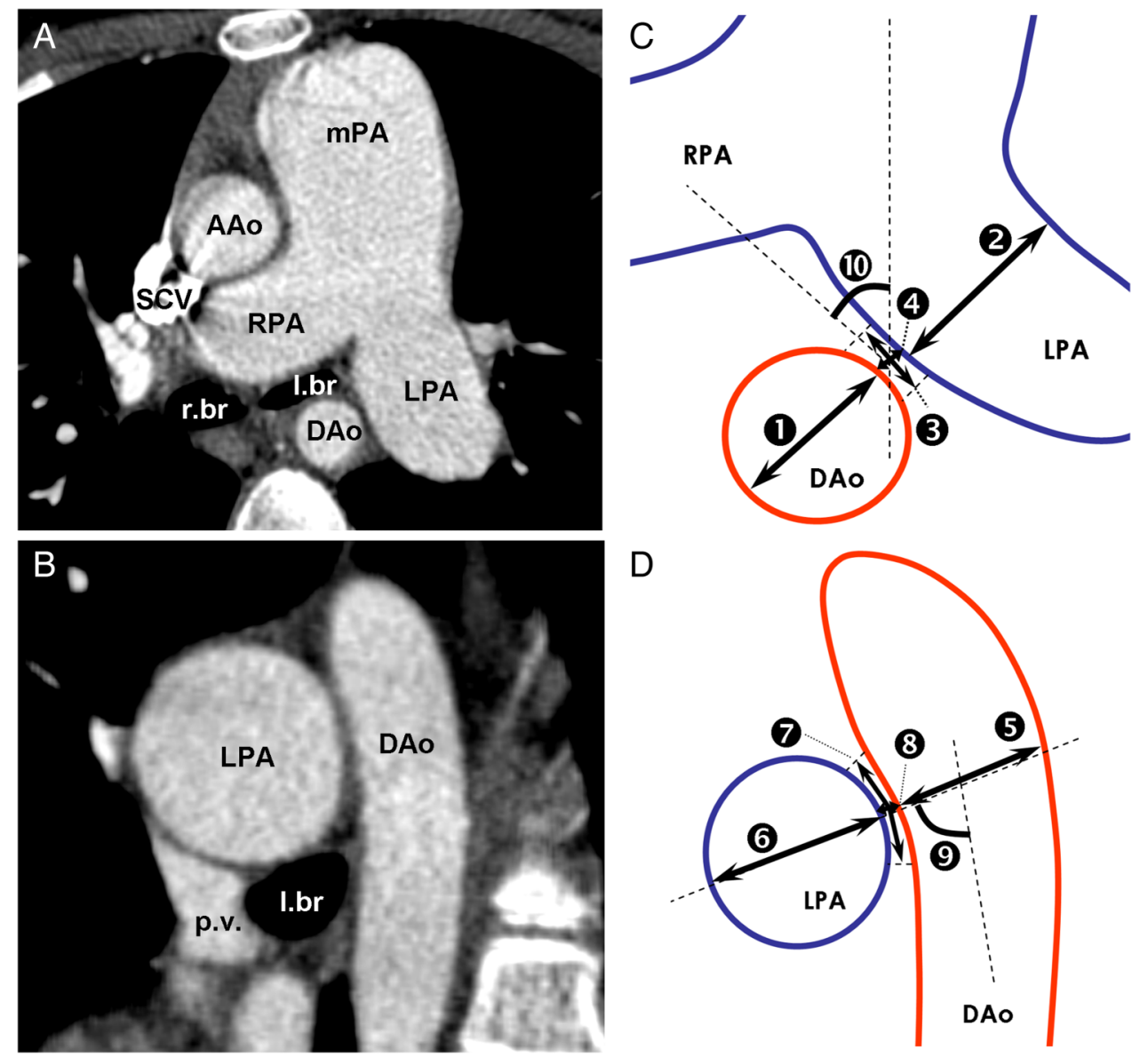

Figure 1 Definition of the morphometric measurements on the CT angiograms. (A and B) show the representative axial and oblique sagittal sections through the descending aorta (DAo) and left pulmonary artery (LPA) in a patient with suprasystemic pulmonary arterial hypertension (PAH). ( $C$ and D) depict the performed measurements by numbers: diameters of LPA ( 2 and 6$)$ and DAo ( 1 and 5$)$; the shortest distance (4 and 8$)$ and the width and height of the elliptic area of the tightest contact ( 3 and 7$)$ between two vessels; the angle between the vertical line and the line perpendicular to the diameter measurements ${ }^{10}$ and the angle between the longitudinal axis of the DAo and the line of the diameter measurements. ${ }^{9}$ 
significant. The intraobserver and interobserver variability was defined as the difference between the two sets of measurements divided by their mean and expressed as percentage per parameter.

\section{RESULTS}

\section{Clinical and haemodynamic data}

Forty-seven patients with 48 thoracic CT angiograms were analysed. One patient with suprasystemic PAH and two CT scans separated by 8 years and accompanied by two catheterisations was included. There were 22 scans from patients with infrasystemic PAH and 26 scans from patients with isosystemic/suprasystemic PAH at the cardiac catheterisation nearest to the CT evaluation (table 1). The age at the CT evaluation was similar for patients with either infrasystemic or isosystemic/suprasystemic PAH (mean \pm SD, $88.9 \pm 59.9$ and $96.3 \pm 58.4$ months, respectively; $\mathrm{p}=0.4$ ). Due to retrospective data collection, $19 \%$ of the scans were performed $>2$ months earlier or later than the nearest invasive haemodynamic evaluation (mean $\pm \mathrm{SD}, 65.3$ \pm 142.3 days; range $1-681$ days). In these patients, echocardiographic data from the moment of CT evaluation was used to confirm the degree of PAH. Patients with isosystemic/suprasystemic PAH had a significantly longer time span between the establishment of diagnosis and the performance of the CT scan (4.6 \pm 9.3 and $14.5 \pm 30.4$ months for the infrasystemic and isosystemic/suprasystemic PAH groups, respectively; $\mathrm{p}<0.01$ ).

\section{Anatomy and morphometry of the region with adjacent descending aorta and LPA}

Table 2 summarises the results of the morphometric measurements. Notably, the average diameters of the DAo and the LPA were very similar in patients with either infrasystemic or isosystemic/suprasystemic PAH. Among 47 scans, there were only 4 scans where the shortest LPA-DAo distance exceeded $3 \mathrm{~mm}$, with three scans from the patients with suprasystemic PAH, making the distance between the two vessels in patients with more severe $\mathrm{PAH}$ significantly longer. However, only one patient had the DAo-LPA distance above $4 \mathrm{~mm}$. In all patients, the DAo wall opposite to the side contacting the LPA was bordered by the vertebral column, while the LPA wall opposite to the side contacting the DAo was bordered by the lung parenchyma. The area of the tightest contact between the two vessels had a weak positive correlation with age and was slightly larger in the patients with infrasystemic PAH. The mean intraobserver

Table 1 Clinical and haemodynamic data

\begin{tabular}{lc}
\hline Age of the patients at CT scan (months) & $92.9 \pm 58.6(2-197)$ \\
Number of scans from patients $<5$ years of age & $40 \%(19 / 48)$ \\
Ratio of PA/systemic systolic pressure & $0.9 \pm 0.3(0.5-1.5)$ \\
Systolic PA pressure (mm Hg) & $87.8 \pm 24.9(50-147)$ \\
Diastolic PA pressure (mm Hg) & $44.9 \pm 16.7(21-86)$ \\
Mean PA pressure (mm Hg) & $62.9 \pm 18.4(36-103)$ \\
Pulmonary capillary (or left atrial or pulmonary venous) & $8.5 \pm 3.3(1-20)$ \\
pressure (mm Hg) & \\
Indexed PVR (Woods $\left.\times \mathrm{m}^{2}\right)$ & $15.3 \pm 7.3(5-41)$ \\
Systolic systemic pressure (mm Hg) & $97.0 \pm 12.3(69-123)$ \\
Diastolic systemic pressure $(\mathrm{mm} \mathrm{Hg})$ & $57.3 \pm 11.0(31-80)$ \\
Mean systemic pressure (mm Hg) & $74.3 \pm 10.2(51-95)$ \\
Indexed cardiac output ( $\mathrm{L} / \mathrm{min} / \mathrm{m}^{2}$ ) & $4.1 \pm 1.1(2.3-7.6)$ \\
\hline Values are mean $\pm S D$ (where appropriate) with the range of minimum and maximum \\
values in brackets. \\
PA, pulmonary artery; PVR, pulmonary vascular resistance.
\end{tabular}

variability was $4.5 \pm 4.4 \%$ for the vessel diameters, $23.7 \pm 18.4 \%$ for the DAo-LPA distance, $13.0 \pm 10.6 \%$ for the width and height of the tightest contact area and $7.6 \pm 7.3 \%$ for the measured angles. The interobserver variability for the abovementioned parameters was $7.1 \pm 5.9 \%, 34.4 \pm 24.1 \%, 34.2$ $\pm 20.6 \%$, and $12.7 \pm 10.6 \%$, respectively. Except for the angles, the variability did not differ significantly for the measurements made on axial and oblique sagittal planes.

No lung tissue was identified between these two vessels in any patient, and no calcification of the vessels was found. The space between the LPA and DAo was bordered anteriorly by the left main bronchus in $70 \%$ of the patients and posteriorly by the lung parenchyma in all patients. The amount of the mediastinal tissue between the two borders varied considerably and did not correlate with any parameter. In four patients $(8.3 \%)$, the prominent bronchial artery was identified coursing exactly between the DAo and LPA (figure 2). Three patients with suprasystemic $\mathrm{PAH}$ and one patient with less severe PAH showed, thus, a high origin of the left-sided bronchial artery that passed between two vessels before entering the left lung hilum. The difference of prevalence of the bronchial arteries between two vessels in both groups did not reach statistical significance.

\section{Spatial relationship and tightest contact area between adjacent descending aorta and LPA}

In $85 \%$ of the analysed scans, the angle of the RAO projection producing the LPA and DAo shadows with the smallest overlay was $\leq 45^{\circ}$, while the angle between the longitudinal axis of the DAo and the line passing through the centres of LPA and the tightest contact area ranged from 75 to $90^{\circ}$. These two angles were very similar for both groups and did not correlate with any parameter. To further illustrate the spatial relationship between the LPA and DAo, the 3D model of these two vessels was prepared based on thoracic CT angiogram of the 7-year-old girl with severe suprasystemic PAH (figure 3A-D). Such relationship between the vessels was representative of the vast majority of the analysed patients, consisting of the immediate vicinity of the proximal LPA to the proximal DAo at relatively short course. Because of the nearly perpendicular angle at which the longitudinal axes of two vessels cross each other and dilation of the LPA, the tightest contact area has an elliptic shape being stretched along the DAo length and LPA width (figure 3E, F). In vast majority of patients, this area differed considerably from the circle, the shape of which would eventually take crosssection of the deployed stent.

\section{DISCUSSION}

Surgical creation of the reversed Potts shunt in a patient with the suprasystemic PAH and failing RV carries high risk of mortality. This justifies development of the less invasive technique to establish the LPA-to-DAo communication. The close proximity of the LPA and DAo creates an attractive opportunity to apply the technique of percutaneous intervascular anastomosis. Several animal experiments ${ }^{18-20}$ and first-in-men reports ${ }^{16}{ }^{17}$ have demonstrated the feasibility of percutaneous perforation of two adjacent vascular walls and deployment of a stent connecting the vessels. For the planning of the transcatheter Potts shunt creation, the comprehensive characterisation of the vascular anatomy is of paramount importance, and has been recently reported for adults with severe $\mathrm{PAH}{ }^{21}$ The systematic evaluation of the anatomic aspects of such a procedure in children with idiopathic PAH is presently lacking. With our study, we sought to fill this gap and provide discussion on the implications of the anatomical findings for the procedural planning. 
Table 2 Morphometric data

\begin{tabular}{|c|c|c|c|c|}
\hline & $\begin{array}{l}\text { Total population; } \\
\mathrm{N}=48\end{array}$ & $\begin{array}{l}\text { Patients with infrasystemic } \\
\text { PAH; } n=22\end{array}$ & $\begin{array}{l}\text { Patients with isosystemicl } \\
\text { suprasystemic } P A H ; n=26\end{array}$ & $\begin{array}{l}\text { Significance of difference } \\
\text { between groups ( } p \text { value) }\end{array}$ \\
\hline LPA diameter (mm) & $16.1 \pm 6.1(5.9-30.5)$ & $15.5 \pm 6.5(5.9-29.9)$ & $16.5 \pm 5.7(8.3-30.5)$ & 0.26 \\
\hline DAo diameter (mm) & $12.2 \pm 2.9(6.6-18.1)$ & $12.3 \pm 3.1(6.6-17.9)$ & $12.0 \pm 2.7(7.9-16.5)$ & 0.48 \\
\hline Shortest distance LPA-DAo (mm) & $1.7 \pm 0.8(1.0-5.0)$ & $1.5 \pm 0.6(1.0-3.7)$ & $1.8 \pm 0.9(1.0-5.0)$ & 0.01 \\
\hline $\begin{array}{l}\text { Area of the tightest contact between } \\
\text { DAo and LPA }\left(\mathrm{mm}^{2}\right)\end{array}$ & $51.8 \pm 31.9(12.5-177.7)$ & $54.9 \pm 33.2(14.1-146.8)$ & $47.4 \pm 24.0(12.5-115.4)$ & 0.05 \\
\hline $\begin{array}{l}\mathrm{CT} \text { scans with identifiable bronchial } \\
\text { arteries between DAo and LPA }\end{array}$ & $8.3 \%(4 / 48)$ & $4.5 \%(1 / 22)$ & $11.5 \%(3 / 26)$ & 0.38 \\
\hline Angle of RAO projection (degrees) & $34.7 \pm 10.6(15-68)$ & $36.3 \pm 11.8(17-68)$ & $33.5 \pm 9.4(17-51)$ & 0.09 \\
\hline $\begin{array}{l}\text { Angle of the curve of catheter tip } \\
\text { (degrees) }\end{array}$ & $85.3 \pm 4.4(75-92)$ & $85.4 \pm 3.8(75-91)$ & $85.5 \pm 4.9(75-92)$ & 0.88 \\
\hline
\end{tabular}

Values are mean \pm SD (where appropriate) with the range of minimum and maximum values in brackets.

DAo, descending aorta; LPA, left pulmonary artery; PAH, pulmonary arterial hypertension; RAO, right anterior oblique.

\section{The intervascular contact area}

During establishment of the connection between the DAo and LPA, the perforation wire using either mechanical force or radiofrequency energy should pass through the adjacent walls of two vessels and the tissue in between. Although the CT angiography provides high-quality imaging of the area of interest, it is hard to assess the thickness of the vessel wall, while its structure and elasticity currently remain undeterminable by this imaging technique. As a result, distance between two contrasted lumens, as we measured, might be overestimated. However, adding the wall thickness to the measured vessel diameters 'reduces' the LPA-to-DAo distance, further facilitating vessel connection and limiting the extravasation risk during the Potts shunt creation. Although efforts were made to standardise the measurements, an oblique nature of CT planes and oval shape of vessel crosssections resulted in a relatively large intraobserver and interobserver variability. However, the variation of the measurements in absolute numbers concerns changes on the (sub)-millimetre scale with not yet known significance for the procedural outcome.

Study on adult patients with severe PAH proposed two types of LPA-DAo arrangement based on the distance and presence of the lung tissue between these vessels. ${ }^{21}$ Interestingly, in our population, we identified only one patient with a LPA-to-DAo distance exceeding the proposed cut-off value of $4 \mathrm{~mm}$. Furthermore, we did not encounter any patient with the lung tissue between the LPA and DAo, which was present in 7\% of adult population with severe $\mathrm{PAH} .^{21}$ Also, no calcifications of
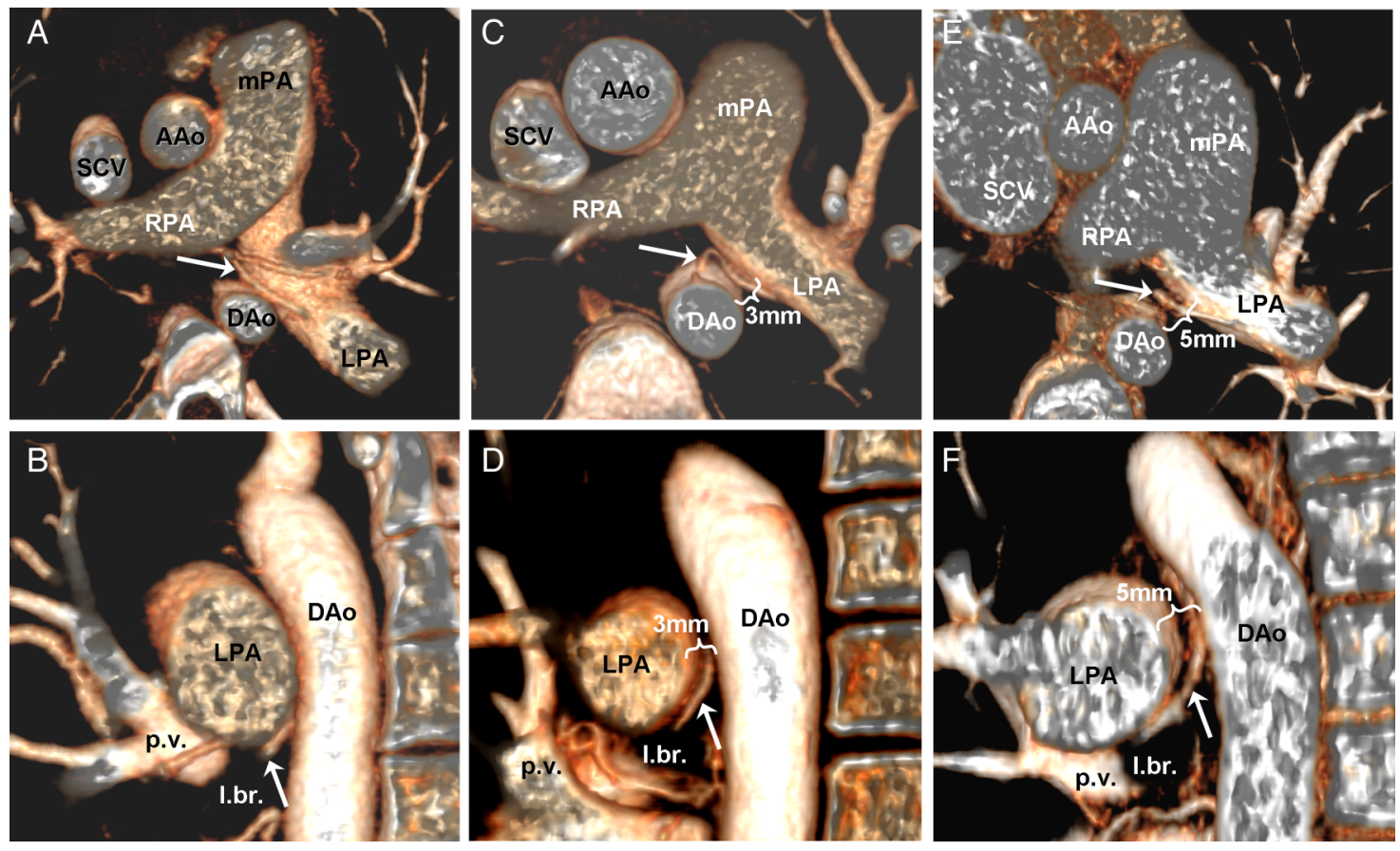

Figure 2 Morphology of the region with adjacent descending aorta and left pulmonary artery (LPA) in three patients with suprasystemic pulmonary arterial hypertension (PAH). These are modified maximum intensity projections in transverse (caudal view, upper panels) and oblique sagittal (left lateral view, lower panels) planes through the LPA and descending aorta (DAo). (A and B) depict the most representative anatomy with the vessels in immediate proximity. Note the prominent bronchial arteries running on the caudal surface of the LPA (arrows) with no structures between two vessels. (C to F) demonstrate two examples of the moderate-to-large distance between the LPA and DAo and clearly identifiable bronchial artery running exactly between the two vessels (arrows). 

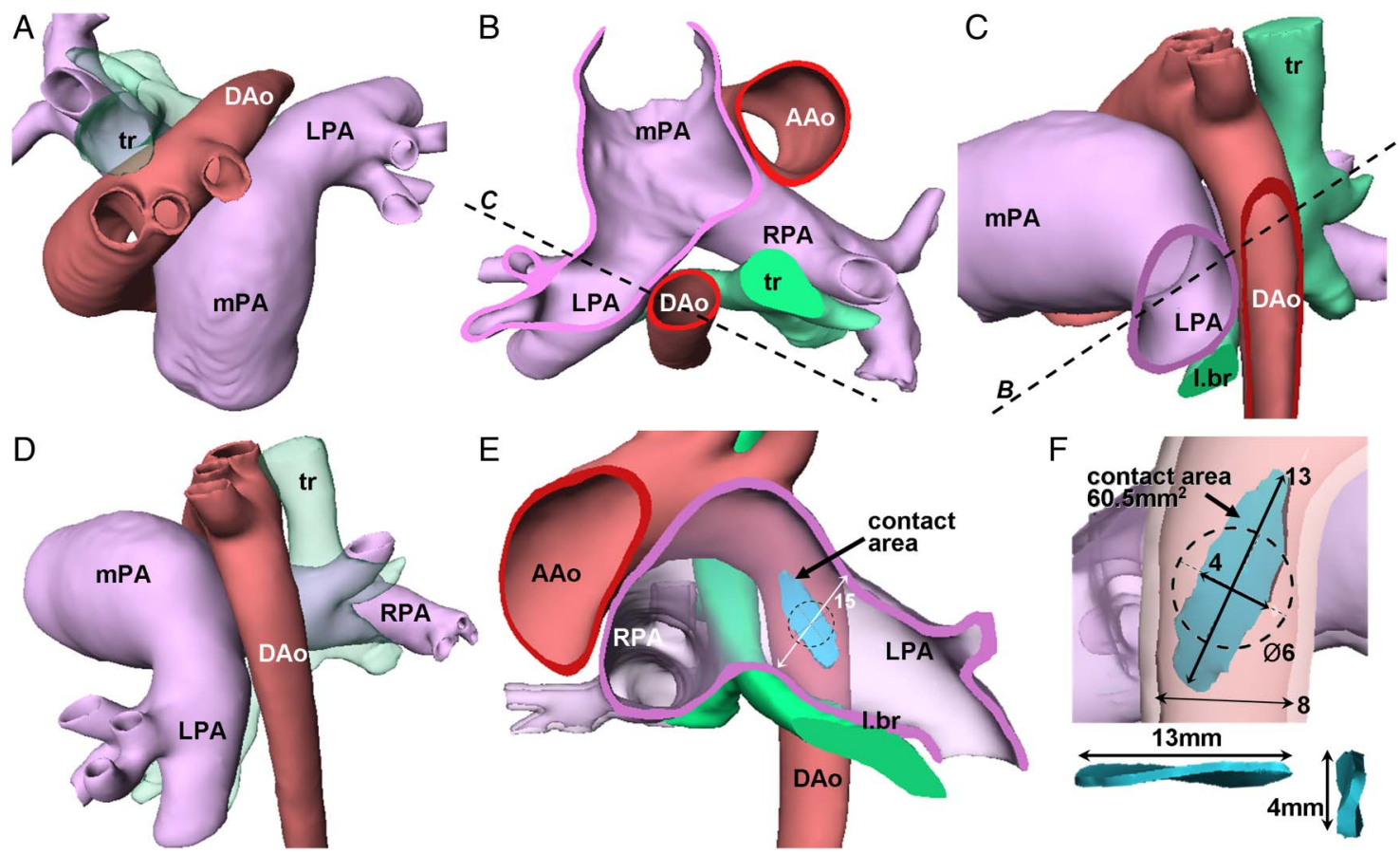

Figure 3 Spatial relationship of the vascular walls in a representative patient with severe suprasystemic pulmonary arterial hypertension (PAH). Aorta is colour-coded as red, pulmonary artery as purple and the trachea-bronchi as light-green. (A) is a cranial view and (D) is the left lateral view of the whole three-dimensional (3D) model. (B) is the craniodorsal view of the transverse cut and $(C)$ is left lateral view of the oblique sagittal cut through the left pulmonary artery (LPA) and descending aorta (DAo). Such arrangement with the very short distance between two vessels was the most prevalent among the population of this study. (E and F) are oblique sagittal cuts through the LPA and DAo, respectively, and show the tightest contact area (colour-coded as light-blue) with the dimensions as indicated (in $\mathrm{mm}$ ). Note the elliptic shape of the tightest contact area with the longest diameter stretched along the DAo length and the LPA width. The dashed circle represents the cross-section of the presumably suitable stent with diameter of $6 \mathrm{~mm}$ being superimposed upon the tightest contact area. Note the differences in the playing space to get the catheter tip at the centre of the tightest contact area in horizontal and vertical planes.

the vessels were found in our paediatric population, compared with $38 \%$ of adults who had aortic calcification at the contact site. ${ }^{21}$ On other hand, we have identified four patients $(8.3 \%)$ in our population with the left bronchial artery running exactly between the LPA and DAo. This circumstance may potentially complicate the procedure by bleeding after unintended damage of the bronchial artery that usually is dilated and prominent in patients with severe $\mathrm{PAH} .^{22}$ One might consider a percutaneous occlusion of the crossing bronchial artery prior to transcatheter creation of the Potts shunt. The preprocedural high-quality CT angiogram is, thus, of crucial importance for the identifying potential complications and planning of the procedure in every individual transcatheter Potts shunt candidate.

\section{Procedural considerations and planning}

The safest location to perform the wire perforation using either mechanical force or radiofrequency energy is through the centre of the area, where these vessels have the tightest contact. Pushing the wire through the vascular walls outside the tightest contact area may result in either entering the lung parenchyma or mediastinal space, or in inconvenient deployment of the stent carrying a risk of dislodgment or vessel damage. When getting the perforation wire from DAo to LPA, there is only one angulation which wire is obliged to take for the safe passage exactly from one vessel into adjacent one. This angle between the DAo longitudinal axis and the line through the centres of LPA and tightest contact area had relatively narrow range in our population. Although this angulation can be achieved using regular cardiovascular catheters with a curved tip, the inevitable modification of the tip curve by the perforating wire stiffness must be taken into account during selection of the delivery catheter. The soft lung parenchyma bordering the LPA wall opposite to the side contacting the DAo might allow the LPA displacement away from DAo either by the delivery catheter passing from one vessel into adjacent one, or by any bleeding between the two vessels. Moreover, an inevitable modification of the intervascular relation and of the final anastomosing stent orientation might be introduced by the stiff exchange wire.

The longest diameter of the DAo-LPA contact area's ellipse is along the DAo length and the LPA width. Such disproportion in the diameters of the contact area results in different playing space for the placement of the catheter tip within the tightest contact area, which may be further challenged by the dynamic nature of the pulsating vessels. While there is a substantial possibility to advance or withdraw the catheter within the DAo (ie, along the height of the ellipse), tip of the catheter can be rotated very little along the DAo circumference (ie, along the ellipse width). The positioning of the catheter tip for the delivery of the perforation wire is of crucial importance and should be thoroughly visualised by angiographic projections. Figure 4 demonstrates the proposed angulations and rotations of the angiographic $\mathrm{C}$-arms derived from the measurements in this study, and resulting views based on the patient-specific 3D anatomy of the vessels. Using the direction of the curved catheter tip within the DAo and appearance of the circle of the open snare within the adjacent LPA in these two orthogonal projections, it should be possible to establish the correct position of the perforation wire tip pointing to the centre of the tightest contact area between the vessels being anastomosed. 

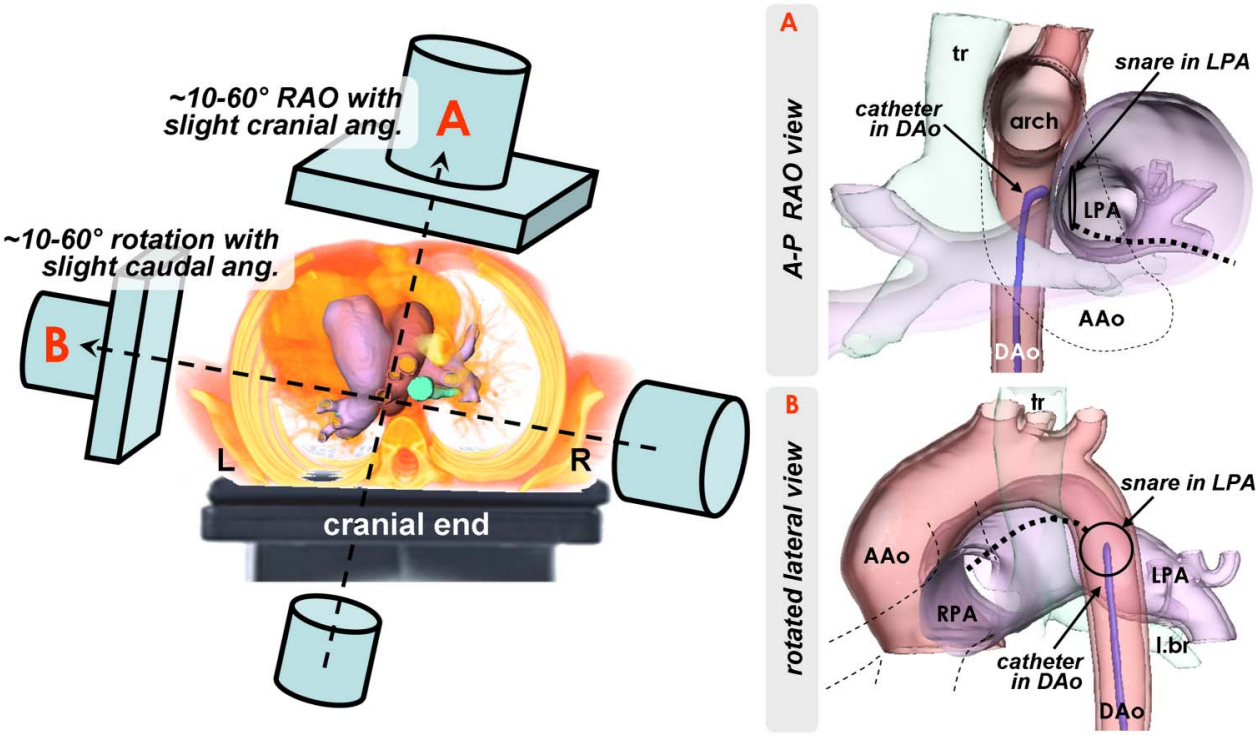

Figure 4 Schematic representation of the $C$-arm positions to produce the oblique anteroposterior $(A)$ and oblique lateral (B) views of the descending aorta (DAo) and the left pulmonary artery (LPA). ( $A$ and B) demonstrate the views produced using the three-dimensional (3D) reconstruction of the patient-specific anatomy of the vessels. The $90^{\circ}$ curved-tip catheter is 'placed' into the DAo with the end pointing to the centre of the tightest contact area between the DAo and LPA (posteriorly and to the patient's left). A ring with dashed line schematically represents a snare 'placed' into the proximal LPA in vicinity of the tightest DAo-LPA contact (to catch the wire upon the perforation of the vessel walls). (A) Right anterior oblique (RAO) view with slight cranial angulation: the adjacent walls of the DAo and LPA are next to each other without overlay; the curved tip of the catheter within the DAo is in profile projection and points towards the centre of the snare within the LPA. Note that the alterations of this RAO projection will foreshorten the curved tip. (B) Counterclockwise rotated lateral view with slight caudal angulation (ie, exactly at $90^{\circ}$ to view $A$ ): the lumens of DAo and LPA are completely superimposed on each other at the area of the tightest contact; the tip of the catheter within the DAo points towards the 'depth' of the screen exactly to the centre of the en face projected snare within the LPA. These two projections should give the visualisation of the course of the wire passing through the adjacent walls of the DAo and the LPA at the centre of the area of the tightest contact between these vessels.

\section{Device considerations}

To achieve the RV afterload reduction, the communication allowing the right-to-left shunting should be unrestrictive. The diameter of the transcatheter stent-secured Potts shunt is limited by the DAo diameter determining the maximal size of the stent that is possible to deploy. The use of a spool-shaped stent, as reported in animals ${ }^{18}$ would be of interest to provide a reduction in the length of the stent, thus, minimising the impairment of aortic flow. The spool-shaped stent with both ends flaring may help to better secure the intervascular anastomosis and to reduce the risk of dislodgement. ${ }^{18}$ Some patients with a Potts shunt indication due to suprasystemic $\mathrm{PAH}$ according to ratio of systolic pressures demonstrate variable PA haemodynamics, especially in the diastole. Further considerations for the dedicated transcatheter Potts shunt device would include the design of the valve mechanism within the stent, similar to the monocusp-like surgical technique. ${ }^{11}{ }^{23}$ Such a valve mechanism will allow only LPA-to-DAo blood flow eliminating potential left-to-right shunting and providing the protection against the higher systemic blood pressure in patients with PAH with variable pulmonary pressures.

\section{Study limitations and conclusions}

Major limitation of our study is performing measurements and assessing intervascular relationship on the static images, which may considerably differ from the situation when heart beats and vessels pulsate. Other limitations are the small number of patients and the retrospective analysis of the data derived from patients where no transcatheter Potts shunt was performed. It does not, thus, allow identification of the anatomic factors playing significant role in the outcome of the procedure.
However, our analysis of the vascular anatomy in substantial number of children with the rare diagnosis of idiopathic PAH showed that, besides the rarely occurring intervascular course of

\section{Key messages}

What is already known on this subject?

Surgical creation of a non-restrictive connection between the left pulmonary artery and descending aorta (Potts shunt) leads to decrease of the right ventricular afterload and to improvement of the symptoms and physical exercise tolerance in children with severe suprasystemic pulmonary hypertension. The thoracic surgery in patients with severe pulmonary hypertension carries high risk of mortality. The percutaneous creation of the stent-secured Potts shunt is feasible and has been reported in adults with severe pulmonary hypertension.

\section{What might this study add?}

Our study provides for the first time the comprehensive analysis of the vascular anatomy in children with pulmonary hypertension regarding the procedure planning of the transcatheter creation of the stent-secured Potts shunt.

\section{How might this impact on clinical practice?}

Understanding of the vascular anatomy and insights into the procedural planning based on the data from our study will add more directed selection of the materials and interventional techniques during the transcatheter creation of the Potts shunt in patients. 
left bronchial artery, there were no complicating anatomic or morphometric parameters potentially determining the transcatheter creation of reverse Potts shunt in paediatric patients with PAH. It holds promises for standardisation of the procedure in the future.

Contributors AS collected and analysed the data and wrote the manuscript. DB and YB participated in the study design and in writing the manuscript. FR participated in the obtaining of the data. All authors read and approved the final manuscript.

Funding AS was supported by the Association pour la Recherche en Cardiologie du Foetus à l'Adulte (ARCFA).

Patient consent Parental/guardian consent obtained.

Ethics approval Local ethical committee of the Necker Children's Hospital, Paris, France.

Provenance and peer review Not commissioned; externally peer reviewed.

\section{REFERENCES}

1 Moledina S, Hislop AA, Foster $\mathrm{H}$, et al. Childhood idiopathic pulmonary arterial hypertension: a national cohort study. Heart 2010;96:1401-6.

2 van Loon RL, Roofthooft MT, Delhaas T, et al. Outcome of pediatric patients with pulmonary arterial hypertension in the era of new medical therapies. Am J Cardiol 2010;106:117-24.

3 Barst RJ, McGoon MD, Elliott CG, et al. Survival in childhood pulmonary arterial hypertension: insights from the registry to evaluate early and long-term pulmonary arterial hypertension disease management. Circulation 2012;125:113-22.

4 Engel PJ, Baughman RP. Treatment of right ventricular dysfunction in pulmonary arterial hypertension: theoretical considerations. Med Hypotheses 2009;73:448-52.

5 Diller GP, Dimopoulos K, Broberg CS, et al. Presentation, survival prospects, and predictors of death in Eisenmenger syndrome: a combined retrospective and case-control study. Eur Heart J 2006;27:1737-42.

6 Hopkins WE, Waggoner AD. Severe pulmonary hypertension without right ventricular failure: the unique hearts of patients with Eisenmenger syndrome. Am J Cardiol 2002;89:34-8.

7 Blanc J, Vouhé $P$, Bonnet $D$. Potts shunt in patients with pulmonary hypertension. N Engl J Med 2004;350:623.

8 Potts WJ, Smith S, Gibson S. Anastomosis of the aorta to a pulmonary artery in certain types in congenital heart disease. J Am Med Assoc 1946;132:627-31.
9 Baruteau AE, Serraf A, Lévy M, et al. Potts shunt in children with idiopathic pulmonary arterial hypertension: long-term results. Ann Thorac Surg 2012;94:817-24

10 Grady RM, Eghtesady P. Potts shunt and pediatric pulmonary hypertension: what we have learned. Ann Thorac Surg 2016;101:1539-43.

11 Baruteau AE, Belli E, Boudjemline $Y$, et al. Palliative Potts shunt for the treatment of children with drug-refractory pulmonary arterial hypertension: updated data from the first 24 patients. Eur J Cardiothorac Surg 2015;47:e105-10.

12 Kula S, Atasayan V. Surgical and transcatheter management alternatives in refractory pulmonary hypertension: Potts shunt. Anatol J Cardiol 2015;15:843-7.

13 Boudjemline Y, Patel M, Malekzadeh-Milani S, et al. Patent ductus arteriosus stenting (transcatheter Potts shunt) for palliation of suprasystemic pulmonary arterial hypertension: a case series. Circ Cardiovasc Interv 2013;6:e18-20.

14 Latus H, Apitz C, Moysich A, et al. Creation of a functional Potts shunt by stenting the persistent arterial duct in newborns and infants with suprasystemic pulmonary hypertension of various etiologies. J Heart Lung Transplant 2014;33:542-6.

15 D'Alto M, Santoro G, Palladino MT, et al. Patent ductus arteriosus stenting for palliation of severe pulmonary arterial hypertension in childhood. Cardiol Young 2015:25:350-4.

16 Esch JJ, Shah PB, Cockrill BA, et al. Transcatheter Potts shunt creation in patients with severe pulmonary arterial hypertension: initial clinical experience. I Heart Lung Transplant 2013;32:381-7.

17 Schranz D, Kerst G, Menges T, et al. Transcatheter creation of a reverse Potts shunt in a patient with severe pulmonary arterial hypertension associated with Moyamoya syndrome. Eurolntervention 2015;11:121.

18 Chigogidze NA, Bilbao Jl, Avaliani MV, et al. Intervascular anastomoses created by an endovascular approach: technical aspects and initial results in an animal study. J Vasc Interv Radiol 2006;17:521-31.

19 Levi DS, Danon S, Gordon B, et al. Creation of transcatheter aortopulmonary and cavopulmonary shunts using magnetic catheters: feasibility study in swine. Pediatr Cardiol 2009;30:397-403.

20 Sabi TM, Schmitt B, Sigler M, et al. Transcatheter creation of an aortopulmonary shunt in an animal model. Catheter Cardiovasc Interv 2010;75:563-9.

21 Guo K, Langleben D, Afilalo J, et al. Anatomical considerations for the development of a new transcatheter aortopulmonary shunt device in patients with severe pulmonary arterial hypertension. Pulm Circ 2013;3:639-46.

22 Devaraj A, Hansell DM. Computed tomography signs of pulmonary hypertension: old and new observations. Clin Radiol 2009;64:751-60.

23 Bui MT, Grollmus O, Ly M, et al. Surgical palliation of primary pulmonary arterial hypertension by a unidirectional valved Potts anastomosis in an animal model. J Thorac Cardiovasc Surg 2011;142:1223-8. 